\title{
Nonlinear pulse combining and compression using twisted hexagonal multi-core fibers
}

\author{
I.S. Chekhovskoy ${ }^{1,2}$, O.V. Shtyrina ${ }^{1,2}$, A.M. Rubenchik ${ }^{3}$, S. Wabnitz ${ }^{1,4}$, M.P. Fedoruk ${ }^{1,2}$ \\ ${ }^{1}$ Novosibirsk State University, Novosibirsk 630090, Russia \\ ${ }^{2}$ Institute of Computational Technologies, SB RAS, Novosibirsk 630090, Russia \\ ${ }^{3}$ Lawrence Livermore National Laboratory, Livermore, California 94550, USA \\ ${ }^{4}$ Dipartimento di Ingegneria dell'Informazione, Elettronica e Telecomunicazioni, Sapienza University of Rome, Rome, Italy
}

\begin{abstract}
We demonstrate numerically and analytically that the twisting of the 7-core hexagonal fiber leads to an increase in the efficiency of pulse combining and to a reduction of the distance along the fiber to the combining point.

Index Terms-multi-core fibers, nonlinear pulse combining,
\end{abstract} nonlinear pulse compression, twisted fibers

Currently, much attention is paid to the study of multicore fibers (MCFs). Despite the fact that the main field of application of these fibers is data transmission in telecommunication lines [1], MCFs have also begun to be used in laser applications [2]. In particular, a promising direction is the study of light focusing using MCFs to create new powerful sources of optical radiation. We have previously shown that the propagation of high-power optical pulses through MCF can lead to energy concentration in a certain core, which leads to coherent combination, amplification and compression of light pulses [3]. This nonlinear effect that occurs in MCFs can be used to create photonic devices such as optical switches, as well as to develop new types of high-power fiber lasers.

In this work we consider a new approach for pulse compression and combining using a MCF twisted relative to the central axis. The addition of uniform twisting qualitatively changes the dynamics of the optical field in MCFs, and allows them to be used to observe a new class of nonlinear effects, for example, PT symmetry breaking.

The propagation of the optical field in a twisted ring MCF with a central core can be described by the following system of coupled NLSEs for the field envelopes:

$$
\left\{\begin{array}{l}
i \frac{\partial U_{0}}{\partial z}=D_{0} \frac{\partial^{2} U_{0}}{\partial t^{2}}+\gamma_{0}\left|U_{0}\right|^{2} U_{0}+\sum_{m=0}^{N} C_{0} U_{m}, \\
i \frac{\partial U_{n}}{\partial z}=D_{n} \frac{\partial^{2} U_{n}}{\partial t^{2}}+\gamma_{n}\left|U_{n}\right|^{2} U_{n}+C_{0} U_{0} \\
+C \exp (-i \phi) U_{n+1}+C \exp (i \phi) U_{n-1}, n=1 . . N .
\end{array}\right.
$$

Here, the parameter $\phi$ determines the amount of twisting relative to the central axis passing along the fiber. To simplify the analysis, all other parameters were set equal to unity.

To study the effect of twisting on the process of nonlinear pulse combining, we considered the best pulse combining regime for a 7-core hexagonal fiber, in which Gaussian pulses $U(t)=\sqrt{P} \exp \left(-t^{2} / \tau^{2}\right)$ with the peak power $P=0.687$ and the width $\tau=1.775$ are simultaneously introduced into

This work was supported by the Ministry of Education and Science of the Russian Federation (Minobrnauka) (14.Y26.31.0017). all cores [3]. The parameter $\phi$ took values from 0 to $\pi / 2$. In the absence of fiber twisting, the point of best combining was determined by the first sufficiently large maximum of the peak pulse power in the central core, which was achieved at $z=1.75$. The combining efficiency was $92.5 \%$.
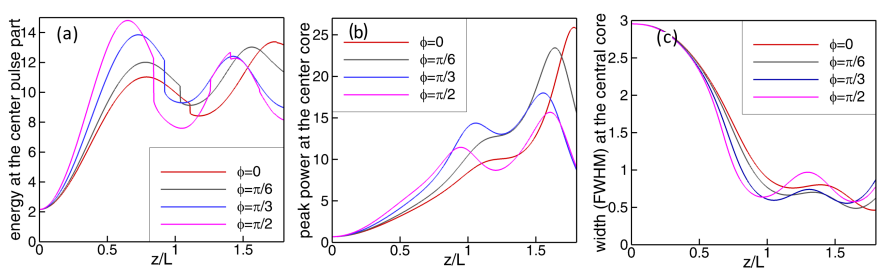

Fig. 1. The dynamics along the fiber of pulse parameters in the central core.

Fig. 1 shows the dynamics along the fiber of various pulse parameters in the central core, in which the combining of all pulses is expected. In Fig. 1(a) the pulse energy excluding energy in the pedestal is shown. As can be seen, with an increase in the twist parameter of the fiber, the pulse energy near the old combining point decreases, although it substantially increased near $z=0.5$. The combining efficiency in this case increases to $99 \%$.

Fig. 1(b) shows the dynamics of the peak power along the fiber, and Fig. 1(c) is the change in pulse width at half maximum (FWHM) in the central core. The maximum value of the peak power decreases in the considered segment of the fiber with an increase in the twist parameter. The minimum value of the pulse width changes slightly.

Summing up, it was shown that the twisting of a 7-core hexagonal fiber leads to an increase in the efficiency of pulse combining, and to reducing the distance along the fiber to the combining point.

\section{REFERENCES}

[1] D. J. Richardson, J. M. Fini, and L. E. Nelson, "Space-division multiplexing in optical fibres," Nat. Photonics 7, 354-362 (2013).

[2] A. A. Balakin, A. G. Litvak, V. A. Mironov, and S. A. Skobelev, "Collapse of the wave field in a one-dimensional system of weakly coupled light guides," Phys. Rev. A 94, 063806 (2016).

[3] I. S. Chekhovskoy, A. M. Rubenchik, O. V. Shtyrina, M. P. Fedoruk, and S. K. Turitsyn, "Nonlinear combining and compression in multicore fibers," Phys. Rev. A 94, 043848 (2016). 